\title{
Resistência do Aedes aegypti ao Temefós em Municípios do Estado do Ceará
}

\author{
Aedes aegypti resistance to temefos in counties of Ceará State
}

\author{
Estelita Pereira Lima ${ }^{1}$, Alfredo Martins de Oliveira Filho², José Wellington de Oliveira Lima ${ }^{3,5}$, \\ Alberto Novaes Ramos Júnior ${ }^{1}$, Luciano Pamplona de Góes Cavalcanti ${ }^{1,4}$ \\ e Ricardo José Soares Pontes ${ }^{1}$
}

\begin{abstract}
RESUMO
Avaliou-se a suscetibilidade de Aedes aegypti ao temefós através de amostras de ovos e larvas procedentes de quatro municípios de grande porte do Estado do Ceará (Fortaleza, Barbalha, Juazeiro do Norte e Crato). Empregou-se a técnica padronizada pela Organização Mundial de Saúde para ensaios com larvicidas. Determinou-se a $C L_{50}$ de oito amostras provenientes de populações de Aedes e suas respectivas razões de resistência, comparada à $C L_{50}$ da cepa suscetível Rockefeller. Todas as populações submetidas ao experimento apresentaram resistência ao temefós, com razões de resistência variando entre 8 e 16. A análise destes resultados reforça evidências anteriores sobre a disseminação de resistência ao temefós em diferentes localidades do estado submetidas a grande pressão de controle nas últimas décadas. O larvicida poderá perder a sua eficácia caso não se busque, com urgência, o restabelecimento da suscetibilidade do Aedes aegypti nestas áreas, afetando sobremaneira as campanhas de controle atualmente em curso.
\end{abstract}

Palavras-chaves: Aedes aegypti. Controle de vetores. Temefós. Resistência a inseticidas.

\begin{abstract}
The susceptibility of Aedes aegypti to temefos was evaluated by means of samples of eggs and larvae from four large counties in the State of Ceará (Fortaleza, Barbalha, Juazeiro do Norte and Crato). The technique standardized by the World Health Organization for tests with larvicides was used. The CL50 of eight samples from populations of Aedes was determined, as were their respective resistance ratios, compared to the CL50 of the susceptible Rockefeller strain. All populations submitted to the experiment showed resistance to temefos, with resistance ratios varying between 8 and 16. Analysis of these results reinforces prior evidence regarding the dissemination of temefos resistance in different locations in the state, subjected to considerable pressure for control in recent decades. The larvicide may lose its effectiveness if an urgent attempt is not made to reestablish the susceptibility of Aedes aegypti in these areas, profoundly affecting control campaigns currently under way.
\end{abstract}

Key-words: Aedes aegypti. Control of vectors. Temefos. Resistance to insecticides.

A crescente incidência do dengue nas últimas décadas e 0 risco de reurbanização da febre amarela requerem estratégias efetivas e eficientes para o enfrentamento desses complexos processos ecológico-epidemiológicos pelos serviços de saúde pública no Brasil. Tradicionalmente, a principal estratégia para o controle do Aedes aegypti tem sido o uso intensivo de inseticidas para a eliminação do mosquito adulto ou de suas larvas. Para o combate ao adulto, utilizam-se com mais frequiência os inseticidas organofosforados e piretróides, geralmente durante as epidemias de dengue. No controle de larvas, o principal larvicida, empregado há décadas no país, é o organofosforado temefós, utilizado a 1ppm de princípio ativo, adsorvido em grãos de areia numa formulação contendo $1 \%$ dessa substância, que pode ser aplicada em água para 0 consumo humano, graças à sua baixa toxicidade oral para mamíferos.

\footnotetext{
1. Departamento de Saúde Comunitária da Universidade Federal do Ceará, Fortaleza, CE. 2. Núcleo de Pesquisas de Produtos Naturais da Universidade Federal do Rio de Janeiro, Rio de Janeiro, RJ. 3. Fundação Nacional de Saúde, Fortaleza, CE. 4. Núcleo de Epidemiologia da Secretaria de Saúde do Estado do Ceará, Fortaleza, CE. 5. Universidade Estadual do Ceará, Fortaleza, CE.

Endereço para correspondência: Prof. Ricardo José Soares Pontes. Deptọ de Saúde Comunitária/Universidade Federal do Ceará. Rua Prof. Costa Mendes 1608/ $5^{\circ}$ andar, Rodolfo Teófilo, 60430-140 Fortaleza, CE.

Tel: 85 4009-80 44, Fax: 85 4009-80 50

e-mail: rjpontes@fortalnet.com.br

Recebido para publicação em 4/1/2005

Aceito em 7/3/2006
} 
0 uso disseminado de substâncias inseticidas pode levar à ocorrência de resistência do mosquito a estes compostos, inviabilizando o controle por essa estratégia de ação. Na realidade, tem-se observado tanto deficiências no controle do A. aegypti determinadas por falhas operacionais de campanha como pela existência de populações de A. aegypti resistentes ao temefós, indicando a necessidade da realização de investigações e de monitoramento da resistência para melhor manejo das ações de controle. 0 fenômeno de resistência do A. aegypti a organofosforados já foi documentado em várias localidades do mundo, tais como: $\mathrm{Cuba}^{14}$, Caribe ${ }^{12}$, Venezuela ${ }^{13}$ e Ilhas Virgens $^{15}$. No Brasil, a resistência a este inseticida já foi detectada em São Paulo $^{6}$, Campo Grande, $\mathrm{MS}^{2}$, Distrito Federal ${ }^{3}$; Rio de Janeiro, Sergipe e Alagoas ${ }^{1}$.

No Estado do Ceará, e no município de Fortaleza o temefós foi utilizado para o controle do A. aegypti desde 1986, quando ocorreu a primeira epidemia de dengue no território cearense $\mathrm{s}^{8}$. Em 2000, foram identificadas algumas amostras de A. aegypti resistentes a este produto em Fortaleza e em Caucaia (região metropolitana de Fortaleza ${ }^{10}$. No final do ano de 2001, o programa de controle do dengue em Fortaleza passou a empregar outro tipo de larvicida, com diferente mecanismo de ação (Bti).
Entretanto, entre 1986 e 2003, ocorreram repetidas epidemias de dengue em Fortaleza e no Estado do Ceará, com uma persistente elevação nos índices de infestação predial, podendo ter ocorrido, como um dos determinantes desse processo, a proliferação de populações de insetos resistentes ao temefós.

0 objetivo desse trabalho foi avaliar, em quatro municípios cearenses, a suscetibilidade de larvas do A. aegypti (único vetor do dengue presente no Estado do Ceará) ao temefós, visando determinar se havia resistência capaz de comprometer o programa de controle e avaliar a dispersão de populações resistentes.

\section{MATERIAL E MÉTODOS}

0 processo de seleção das áreas estudadas foi estabelecido considerando-se inicialmente a identificação de áreas metropolitanas e pólos econômicos do Estado do Ceará, historicamente sob grande pressão de controle químico (especialmente o larvicida temefós) e com elevados índices de infestação predial nos dois anos anteriores à pesquisa, realizada em 2003. Assim, foram selecionados para o estudo os municípios de Fortaleza, Barbalha, Crato e Juazeiro do Norte (Figura 1).

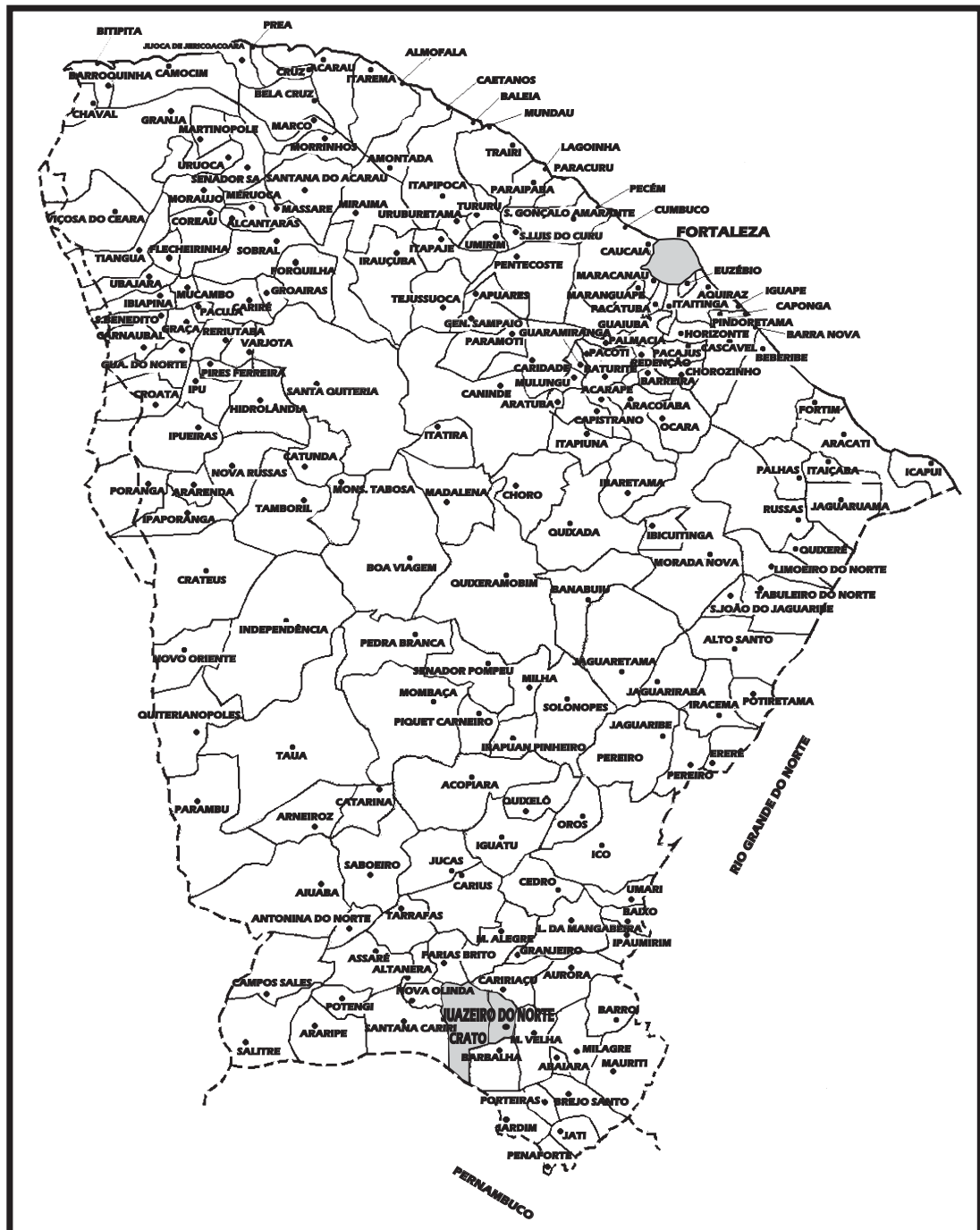

Figura 1 - Mapa do Estado do Ceará com a localização geográfica dos municípios de Fortaleza, Juazeiro do Norte e Crato, 2003. 
0 município de Fortaleza é dividido administrativamente em seis secretarias regionais e, desta forma, foram instaladas ovitrampas em dez bairros distribuídos entre as seis regionais, buscando uma representação geográfica intencional de toda a área, ou quadrantes, do município (Regional I: Bairro de Jacarecanga; II: Joaquim Távora; III: Bom Sucesso e Rodolfo Teófilo; IV: Demócrito Rocha e Damas; V: Granja Portugal, Vila Manoel Sátiro e Parque São José; VI: Jangurussú) (Figura 2).

Nos outros municípios do interior, localizados a mais de $500 \mathrm{~km}$ de Fortaleza (Figura 1), no extremo sul do Estado do Ceará, foram coletadas larvas em criadouros domiciliares procedentes dos bairros: Vila Santo Antônio, em Barbalha; João Cabral e Casas Populares, em Juazeiro do Norte; e Centro, em Crato.

As amostras de ovos provenientes de Fortaleza foram levadas ao laboratório e identificadas para o estabelecimento de colônias e obtenção da geração F1. Nos municípios do interior do Estado, as larvas coletadas no campo foram selecionadas e identificadas para a formação de colônias e obtenção da primeira geração de larvas em laboratório. Os bioensaios foram realizados com larvas de $3^{\circ}$ estádio da referida geração. Em cada teste foram expostos três lotes de 25 larvas em $250 \mathrm{ml}$ de água, na concentração de 0,012ppm de temefós, correspondente à concentração diagnóstica desse produto para $A$. aegypti, preconizada pela Organização Mundial de Saúde ${ }^{1619}$. 0 controle foi realizado expondo-se
25 larvas a $1 \mathrm{ml}$ de acetona em $250 \mathrm{ml}$ de água. Simultaneamente, realizou-se um teste com larvas comprovadamente suscetíveis da linhagem Rockefeller, cedida pelo Núcleo de Pesquisas de Produtos Naturais da Universidade Federal do Rio de Janeiro, expondo-as à concentração de 0,012ppm de temefós.

A leitura dos testes foi realizada após 24 horas, calculandose a taxa de mortalidade das larvas. Considerou-se como indicador qualitativo de resistência ao larvicida (presente ou ausente) quando a mortalidade ficou abaixo de $80 \%$. Nessa classificação, seria suscetível quando esse percentual de mortalidade fosse acima de $98 \%$, sendo os valores entre 80 e $98 \%$ inconclusivos, necessitando acompanhamento ${ }^{4}$. Os sobreviventes à concentração diagnóstica indicaram a possibilidade da presença de indivíduos portadores do gene ou dos genes para resistência ao temefós na população de A. aegypti de cada bairro estudado. Os grupos de larvas que sobreviviam a esta concentração foram submetidos a concentrações crescentes para se determinar a $\mathrm{CL}_{50} \mathrm{e}$ posteriormente a razão de resistência (RR) ao temefós. Para tanto, utilizou-se o programa SPSS for Windows (versão 10.0) para a análise de regressão log probit considerando-se pelo menos três percentuais de mortalidade entre $10 \%$ e $90 \%{ }^{5}$. A RR dos grupos de larvas expostos foi determinada dividindose o valor da $\mathrm{CL}_{50}$ das larvas resistentes pela $\mathrm{CL}_{50}$ da linhagem suscetível Rockefeller.

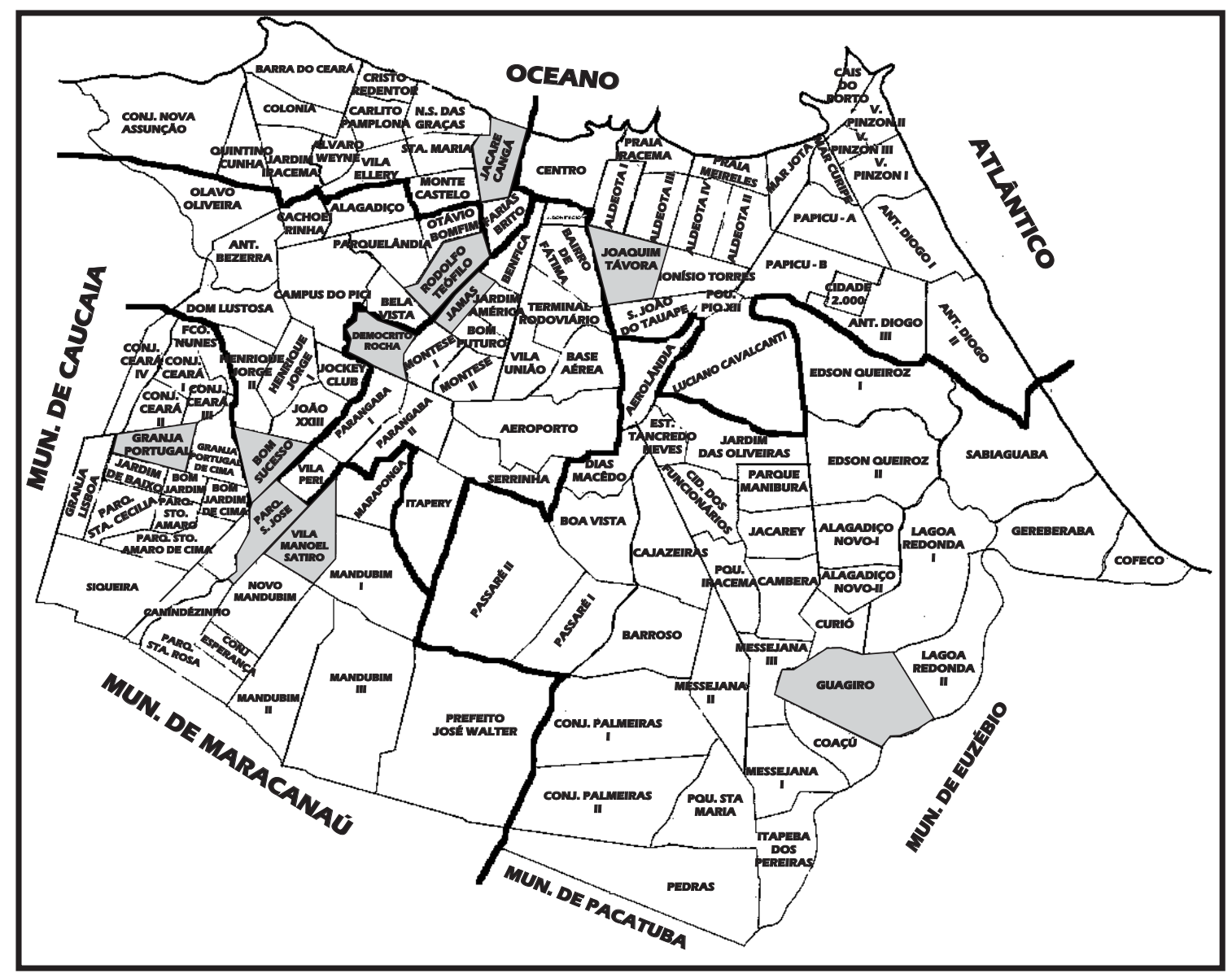

Figura 2 - Mapa do Município de Fortaleza com a divisão por Secretarias Executivas Regionais e bairros amostrados, 2003. 


\section{RESULTADOS}

As amostras de A. aegypti provenientes dos bairros de Fortaleza apresentaram um baixo percentual de mortalidade quando expostas ao temefós, sempre em patamares inferiores a $80 \%$ (a mortalidade em cada Secretaria Regional de Saúde variou de um mínimo de 0 a um máximo de $28 \%$ ), valores fortemente sugestivos da presença de indivíduos resistentes nessa população. Em mais da metade dos bairros estudados, a taxa de mortalidade foi nula ( $0 \%)$, confirmando essa tendência. Foi detectada resistência uniformemente em todas as seis Regionais de Fortaleza, revelando a dispersão desse caráter por toda a área metropolitana da cidade (Tabela 1).

Da mesma forma, reduzidos percentuais de mortalidade (de $0 \%$ a $4 \%$ ) foram também detectados em $A$. aegypti procedentes dos municípios de Juazeiro do Norte, Crato e Barbalha, evidenciando que a resistência mostra-se também difundida no interior sul do Estado (Tabela 1).

Testes posteriores para determinar a razão de resistência dos grupos de larvas sobreviventes revelaram níveis elevados de resistência, tanto em Fortaleza quanto nos municípios do interior sul do estado. Em apenas um grupo de larvas de Fortaleza a razão de resistência (RR) ao temefós encontrava-se abaixo de 10. Os demais apresentaram RR entre 10 e 16, valores considerados elevados, capazes de interferir na eficácia do controle. Nos grupos de larvas coletados em Juazeiro do Norte, Barbalha e em Crato, todas as RR encontravam-se acima de 10 (Tabela 2).

Tabela 1 - Mortalidade de larvas (\%) em populações de Aedes aegypti expostas à concentração diagnóstica do temefós $(0,012 \mathrm{ppm})$, provenientes de quatro municípios do Estado do Ceará, 2003.

\begin{tabular}{lcccc} 
Municípios & $\begin{array}{c}\text { Larvas expostas } \\
\left(\mathrm{n}^{0}\right)\end{array}$ & $\begin{array}{c}\text { Vivas } \\
\left(\mathrm{n}^{0}\right)\end{array}$ & $\begin{array}{c}\text { Mortas } \\
\left(\mathrm{n}^{0}\right)\end{array}$ & $\begin{array}{c}\text { Mortalidade } \\
(\%)\end{array}$ \\
\hline Juazeiro do Norte & 75 & 75 & 0 & 0,0 \\
& 75 & 74 & 1 & 1,3 \\
\hline Crato & 75 & 72 & 3 & 4,0 \\
\hline Barbalha & 75 & 71 & 4 & 5,3 \\
\hline Fortaleza (Regionais de Saúde) & & & & \\
I & 75 & 71 & 3 & 4,0 \\
II & 75 & 75 & 0 & 0,0 \\
III & 75 & 63 & 12 & 16,0 \\
III & 75 & 75 & 0 & 0,0 \\
IV & 75 & 75 & 0 & 0,0 \\
IV & 75 & 54 & 21 & 28,0 \\
V & 75 & 66 & 9 & 12,0 \\
V & 75 & 75 & 0 & 0,0 \\
V & 75 & 75 & 0 & 0,0 \\
VI & 75 & 75 & 0 & 0,0 \\
\hline
\end{tabular}

Tabela 2 - Concentrações Letais $\mathrm{CL}_{50}$, limites de confiança (95\%) e razões de resistência $(\mathrm{RR})$ ao temefós de amostras de Aedes aegypti coletadas em Fortaleza, Juazeiro do Norte e Crato-CE, em 2003*.

\begin{tabular}{lccc}
\hline Procedência das amostras & $\mathrm{CL}_{50}$ & Limite & $\mathrm{RR}$ \\
\cline { 3 - 3 } Municípios & & inferior - superior & \\
\hline Fortaleza & 0,026 & $(0,024-0,028)$ & 10 \\
& 0,037 & $(0,036-0,039)$ & 15 \\
& 0,021 & $(0,019-0,024)$ & 8 \\
& 0,040 & $(0,038-0,041)$ & 16 \\
& 0,027 & $(0,025-0,030)$ & 11 \\
\hline Juazeiro do Norte & 0,033 & $(0,030-0,036)$ & 13 \\
& 0,028 & $(0,025-0,031)$ & 11 \\
\hline Crato & 0,033 & $(0,031-0,035)$ & 13 \\
\hline Rockfeller (Colônia Suscetível) & 0,0025 & $(0,002-0,002)$ & 1 \\
\hline
\end{tabular}

* Dados das populações em que o programa SPSS foi adequado à análise.

\section{DISCUSSÃo}

Os resultados do presente estudo mostraram fortes evidências de que as amostras de A. aegypti coletadas nos bairros de Fortaleza encontravam-se resistentes ao larvicida temefós, indicando, pela grande abrangência geográfica, que provavelmente esta resistência já havia se estabelecido há vários anos. A resistência mostrou-se dispersa em todas as regionais de Fortaleza, provavelmente, pela pressão de controle ter sido uniformemente distribuída em todo o município e devido à inexistência de barreiras geográficas capazes de impedir o cruzamento entre estas populações. Os atuais resultados comprovaram os achados iniciais de Oliveira Filho e cols ${ }^{10}$, em 2001, quando identificaram amostras de A. aegypti resistentes ao temefós em áreas restritas e localizadas da grande Fortaleza ${ }^{10}$. No presente estudo, mesmo utilizando-se uma amostra menor de larvas que o usualmente recomendado ${ }^{17} 18$ e aplicando-se uma metodologia, que, segundo Braga e cols ${ }^{1}$, tenderia a subestimar a detecção desse fenômeno, observou-se que a resistência era de importante magnitude e dispersão. Esse achado é um indicador indireto de que a prevalência da ocorrência de resistência deve ser elevada nas realidades estudadas, pois foi possível detectá-la mesmo com pequenas amostras, sugerindo a gravidade da situação em termos epidemiológicos ou de ações de prevenção e controle. A presença de amostras resistentes nos municípios do interior do Estado encontrava-se tão alta quanto à observada em Fortaleza. Considerou-se esse achado como importante indicador da presença de populações de A. aegypti resistentes em outros municípios que sofreram forte pressão de seleção pelo temefós, fato que merece atenção em virtude do uso disseminado e prolongado desse produto em quase todo o Estado.

Além da baixa mortalidade frente ao temefós, indicativa da forte presença de indivíduos resistentes, o fato mais preocupante foi 0 alto patamar das razões de resistência (RR) a esse larvicida, nas populações testadas. Essas se apresentaram, em sua maioria, com RR acima de 10, nível considerado comprometedor da ação do larvicida, causando prejuízos aos programas de controle do vetor?. Para larvas 
que apresentam RR desta magnitude, o efeito residual do temefós em campo será menor, uma vez que há queda gradual da concentração disponível inicialmente nos depósitos tratados, contribuindo para o aumento no número de larvas sobreviventes com o passar do tempo, com o conseqüente incremento da infestação vetorial nas respectivas áreas. Esta situação acarreta a necessidade de maior número de aplicações de larvicida em intervalos mais curtos, com 0 conseqüente aumento de custos no programa de controle e riscos de ocorrência de epidemias de dengue.

Diante das condições de resistência das populações testadas, coloca-se a necessidade de substituição do temefós por outros larvicidas com mecanismo de ação diferente, visando maior efetividade do programa de controle e a recuperação da suscetibilidade da espécie alvo ao produto anteriormente em uso. Da mesma forma, reforça-se a fundamentação científica sobre a decisão de substituir este larvicida (o temefós) pelo Bti, nos municípios de Fortaleza (em 2001), Juazeiro do Norte e Caucaia (em 2002). No entanto, nas outras duas cidades, Crato e Barbalha, além dos demais municípios do Ceará, o temefós ainda continuava sendo aplicado, aumentando a pressão seletiva por esse organofosforado e a probabilidade de elevar cada vez mais os níveis de resistência das populações de $A$. aegypti lá existentes.

Além da substituição do larvicida, sugere-se ainda que se invista em formas de controle alternativas que possam eliminar ou minimizar os riscos de desenvolvimento de resistência aos produtos utilizados. Uma delas é o controle integrado, medida que está sendo incentivada no Brasil, envolvendo o controle químico, biológico e mecânico, com a participação da população e respeitando as peculiaridades de cada área infestada. Dessa forma, estratégias de educação ambiental e educação em saúde devem, necessariamente, ser desenvolvidas. Também é indispensável que haja investimento no sistema de saneamento básico, coleta de lixo e outras formas de manejo urbano que minimize o risco representado por criadouros de $A$. aegypti ${ }^{9}$.

A adoção do controle integrado, inclusive com o uso de peixes larvófagos em depósitos domiciliares, é uma prática que já vem sendo largamente utilizada no Estado do Ceará ${ }^{11}$. Diante do quadro de resistência das populações de A. aegypti avaliadas é preciso que seja intensificado esse tipo de controle, como também, proceder a avaliação de amostras de populações de $A$. aegypti em outras localidades, além do monitoramento permanente dos produtos utilizados nas ações de controle do vetor.

\section{AGRADECIMENTOS}

À bióloga Ana Cláudia Regazzi e aos laboratoristas Zolide Ribeiro, José Maria e Antônio Raimundo da Fundação Nacional de Saúde; ao grupo do Núcleo de Pesquisas de Produtos Naturais da Universidade Federal do Rio de Janeiro e aos agentes de endemias dos municípios de Fortaleza, Juazeiro do Norte, Crato e Barbalha.

\section{REFERÊNCIAS BIBLIOGRÁFICAS}

1. Braga IA, Lima JBP, Soares SS, Valle D. Aedes aegypti resistance to temephos during 2001 in several municipalities in the states of Rio de Janeiro, Sergipe, and Alagoas, Brazil. Memórias do Instituto Oswaldo Cruz 99: 199203,2004 .

2. Campos J, Andrade CFS. Susceptibilidade larval de populações de Aedes aegypti e Culex quinquefasciatus a inseticidas químicos. Revista de Saúde Pública 37:523-527, 2003.

3. Carvalho MSL, Caldas ED, Degallier N, Vilarinhosa PTR, Souza LCKR Yoshizawa MAC, Knox MB, Oliveira C. Suscetibilidade de larvas de Aedes aegypti ao inseticida temefós no Distrito Federal. Revista de Saúde Pública 38:623-629, 2004

4. Davidson G, Zahar AR. The Pratical implications of resistance of malaria vectors to insecticides. Bulletin Word Health Organization 49: 475-483, 1973.

5. Finney DJ. Probit analysis. A statistical treatment of the sigmoid curve. Cambridge University Press Cambridge, England, 1964.

6. Macoris MLG, Andrighetti MTM, Takaku L, Flasser C, Garbeloto VC, Cirino CB. Alteração de resposta de suscetibilidade de Aedes aegypti a inseticidas organofosforados em municípios do Estado de São Paulo, Brasil. Revista de Saúde Pública 33:521-522, 1999.

7. Mazzari MB, Georghiou GP. Characterization of resistence to organophosphate, carbamate, and pyrethroid insecticides in field populations of Aedes aegypti from Venezuela. Journal American Mosquito Control Association, 11: 315-322, 1995.

8. Nogueira MB. Infestação pelo Aedes aegypti em Fortaleza no período de 1986 a 1998: Estratificação de Risco. Dissertação de Mestrado, Universidade Federal do Ceará, Fortaleza, CE, 1999

9. Oliveira Filho AM. A resistência dos insetos aos inseticidas e o controle dos vetores da malária. In: Mariconi AM (ed) Insetos e outros invasores de residências. Fundação de Estudos Agrários Luiz de Queiroz, São Paulo, p. 379-402, 1999.

10. Oliveira Filho AM, Costa EG, Melo MTV, Santos CE, Griffo HG, Lustosa ELB, Ribeiro ZM. Resistência a Temefós em linhagens de Aedes aegypti provenientes do Ceará. In: Resumos do XXXVII Congresso da Sociedade Brasileira de Medicina Tropical, Salvador p. 291, 2001.

11. Pamplona LGC, Lima JWO, Cunha JCL, Santana EWP. Avaliação do impacto na infestação por Aedes aegypti em tanques de cimento do município de Canindé, Ceará, Brasil, após a utilização do peixe Betta splendens como alternativa de controle biológico. Revista da sociedade Brasileira de Medicina Tropical 37: 400-404, 2004

12. Rawlins SC, Wan JOH. Resistance in some Caribbean populations of Aedes aegypti to several insecticides. Journal of the American Mosquito Control Association 11:59-65, 1995.

13. Rodrigues MM, Bisset J, Fernandez DM, Lauzán L, Soca A. Detection of insecticide resistance in Aedes aegypti (Diptera, Culicidae) from Cuba and Venezuela. Journal of Medical Entomology 38:623-628, 2001.

14. Rodrigues MM, Bisset JA, Milá LH, Calvo E, Diáz C, Soca LA. Niveles de resistencia a insecticidas y sus mecanismos en una cepa de Aedes aegypti de Santiago de Cuba. Revista Cubana de Medicina Tropical 51:83-88, 1999.

15. Wirth MC, Georgiou GP. Selection and characterization of temephos resistance in a population of Aedes aegypti, from Tortola, British Virgin Islands. Journal of the American Mosquito Control Association 15:315-320, 1999.

16. Word Health Organization. Insecticide Resistance and Vector Control. WHO Technical Report Series, Geneva, 1970.

17. Word Health Organization. Instructions for Determining the Susceptibility or Resistance of Mosquito Larvae to Insecticides. WHO/VBC/81.807, Geneva, 1981.

18. Word Health Organization. Criteria and Meaning of Tests for Determining the Susceptibility or Resistance of Insects to Insecticides. WHO/VBC 81.16, Geneva, 1981

19. World Health Organization. Vector resistance to pesticides: fifteenth report of the WHO Expert Committee on Vector Biology and Control. WHO Technical Report Series, 818, Geneva, 1992. 